\title{
La universidad, la escuela y el museo: relaciones e implicaciones educativas desde la mirada de Teresa Torres de Eça
}

Mạ Dolores SOTO-GONZÁLEZ. Florida Universitària. València (España). Isoto@ florida-uni.es

Esta entrevista a Teresa Torres Eça fue realizada el sábado 30 de Marzo de 2017.

El lugar, el Museo da Quinta da Cruz de Viseu sede de Associação de Professores de Expressão e Comunicação Visual- APECV/ www.apecv.pt. Los días compartidos con Teresa han sido excepcionales. Amante de las artes y de la educación, no hubo ningún problema por su parte en contestar las preguntas realizadas, que tuvieron lugar en diferentes momentos ya que estar con Teresa es viajar, compartir y vivir.

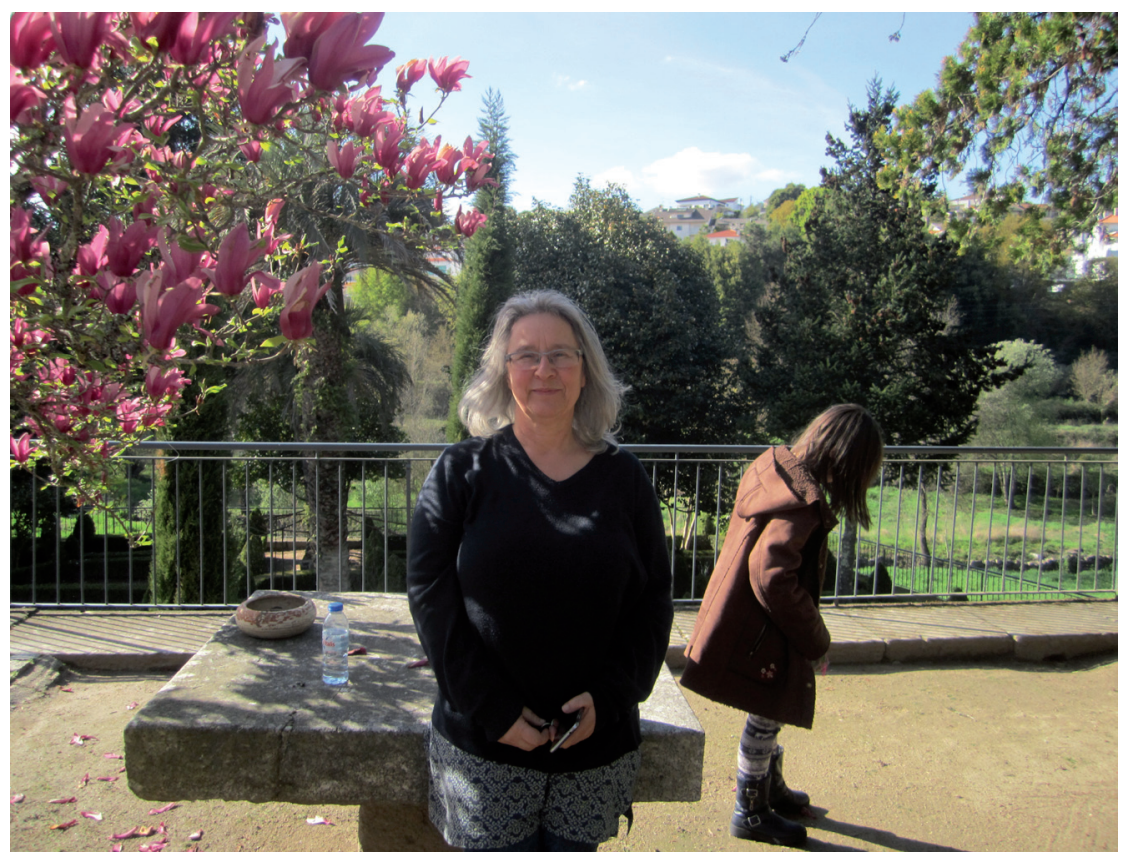

Figura 1. Teresa Eça con su hija en los jardines del Museo Quinta da Cruz (Viseu-Portugal) donde se encuentra la Sede de la Asociación de Profesores de Expresión y Comunicación Visual - AEPCV que preside. 
Teresa Torres de Eça es Presidenta da Associação de Professores de Expressão e Comunicação Visual- APECV/ www.apecv.pt y President of The International Society For Education Throught Art- InSEA. www.insea.org

LSG: ¿Cómo vives el hecho de ser una de las personalidades más importantes en el panorama internacional de la Educación Artística como Presidenta del InSEA?

TTE:Yo no creo que sea una de las personalidades más importantes, sino que tengo mucha facilidad de relacionarme con el otro. Escucho a mucha gente y entonces la gente me ha elegido, no por ser importante sino por la manera como las escucho. Han visto en mí una persona que sabe articular, que hace conexiones con las organizaciones, las instituciones y las personas. Creo que no es por ser importante, es por ser "social skill". Cuando me propusieron para ser presidenta, primero dije que no, pero por la insistencia desde InSEA me presenté. Luego votaron y aquí estoy, muy sorprendida porque soy de un país periférico. No tengo el poder de una Universidad como normalmente se tiene, no trabajo en una universidad formalmente. Y tal vez sea por eso, por ser una persona diferente y agregadora, me han elegido por eso, no por ser importante. Por agregar a las personas, a las ideas y conectarlas. Hay una cosa que he aprendido a hacer con el tiempo que es sacarlo que la gente tiene de bueno, quizás sea uno de los factores que han hecho de mí lo que soy hoy.

LSG: La clave de la presencia en el mundo de educación artística sigue siendo InSEA, organización de la que eres presidenta mundial.

TTE: Sí, creo que poco a poco, InSEA se está reafirmando como una organización que está siendo llamada y consultada para dar su opinión. Por ejemplo, en la UNESCO ahora hay una reunión con una delegación de Europa, del Consejo Europeo que está trabajando el currículum y han llamado a InSEA para que vaya a dar su opinión. Sí, poco a poco, por ejemplo en América Latina, África, en países de centro Europa, InSEA tiene un papel muy importante, en la defensa de la Educación Artística.

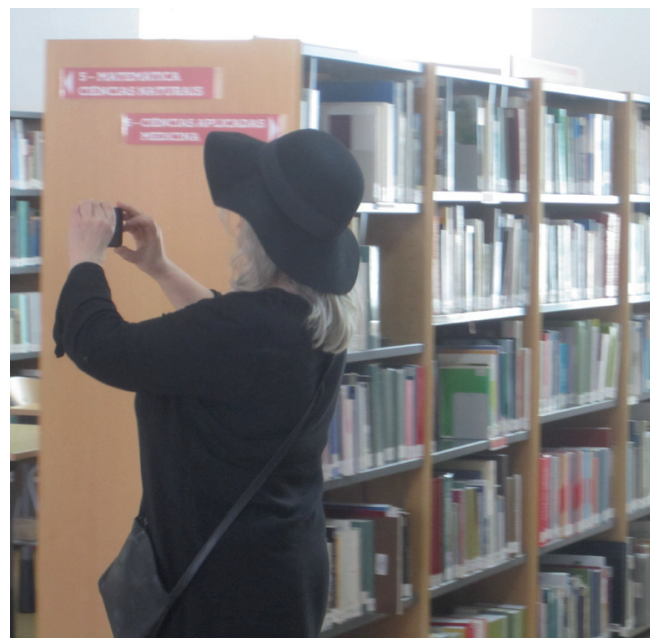

Figura 2. Teresa Torres de Eça en la Biblioteca de Bragança fotografiando la exposición de Cadernos Artivistas el día de su inauguración, sábado 8 de Abril del 2017. 


\section{LSG: ¿Cuál es el estado de la educación artística en la escuela de hoy?}

TTE: En la escuela formal, ¿qué educación artística? Primero tenemos que hablar de lo que se entiende por educación artística. Si entendemos educación artística como instrucción, como la enseñanza de asignaturas relacionadas con arte o con cultura visual, o con tecnologías visuales, pues sí que hay. Pero será eso lo que entendemos nosotros por educación artística. Yo creo que la educación artística que nosotros queremos, no existe en las escuelas o existe muy poco. Creo que debe ser una educación artística emancipadora, habrá gente que trabaja con los temas de la sociedad y que utiliza el lenguaje artístico como expresión sobre el mundo. Pero eso se ve muy poco, lo que veo más es instrucción, es enseñanza de las artes, no propiamente educación artística. Siempre hay esa manera de ver esa educación artística en la escuela, aquí en Portugal se llama educación visual, en España se llama educación plástica, o en Francia plástica o art education. Se ve siempre desde el punto de vista del artista y no del ciudadano, no se ve como comunicación. Se dice, vamos a aprender como los artistas hacen, vamos a aprender, vamos a apreciar obras de arte, vamos a hacer obras de arte, pero no se dice vamos a pensar con herramientas artísticas, y hay que seguir luchando por eso, por el pensamiento.

\section{LSG: ¿Cómo definirías el concepto o término arte-educación? ¿Por qué hablamos de arte + educación? ¿Desde cuándo se comenzó a hablar de la educación a través del arte?}

TTE: (Se ríe). Educación a través del arte es InSEA, desde el inicio, es toda una ideología de la postguerra que la educación a través del arte podría traer más paz al mundo y la gente sería más sensible. Pero si nos paramos a pensar, a veces los artistas no son más pacíficos, no son más solidarios, por eso no se puede decir que el arte va a traer consigo valores. El arte como el arte en sí, es un medio de comunicar, de transmitir, pero cuando se habla de educación, se habla de valores, de ciudadanía, de transformación social; Y preparar el futuro, es importante en la educación preparar el futuro. Es desde los 50' que se habla de educación por el arte, pero no existe, lo que vemos es todo con las disciplinas, las asignaturas, la estética, la crítica de arte, la producción artística (hace referencia a Eisner). Para mí la educación es educación con las artes, no se dice educación para la literatura, no se dice educación para la matemática, nadie pensaría en decir eso. No vamos a ser escritores pero vamos a utilizar la literatura para que te construyas como individuo, lo mismo ocurre con el arte. Ahora, la educación por el arte no sé, es el arte en la educación, como la literatura en la educación. El arte es un campo del conocimiento muy importante de la humanidad, porque no llamarle "arte", mejor "artes" que son varias.

LSG: ¿Qué claves ofrece el arte a la educación? ¿Qué puede seguir aportando el arte a la educación? 
TTE: Otra manera de representar el mundo, otra manera de pensar, una lógica muy próxima a lógica científica pero distinta; Porque es el pensamiento artístico, la resolución de problemas artísticos, es diferente, aunque su significado puede ser muy parecido. El arte tiene sus propios procesos para ayudar a la gente a ser representada, a construirse, a relacionarse con el otro.

LSG: ¿Debe enseñarse el arte por su enfoque histórico o por las temáticas, géneros, significaciones etc.?

TTE: Debe enseñarse por las cuestiones, cualesquiera que sean las cuestiones. Si quieres trabajar la violencia de género tienes toda la pintura barroca, está todo interconectado.

LSG: ¿Qué pueden aportar organizaciones e instituciones como museos, fundaciones, incluso ferias... al aprendizaje no sólo del arte y de la expresión artística sino al contexto educativo en general?

TTE: Información, son recursos. Yo trabajo mucho con museos, centros culturales, con todo, con todo lo que aparezca. Creo que esas instituciones están ahí, que son recursos que deben estar abiertos. El problema es como tú trabajas los recursos, porque hay de todo. Por ejemplo, hay museos que te ofrecen muchísimas cosas pero con una ideología por detrás, que es la ideología del museo, de promoción de la colección y una manera de ver también la educación, muy propia. A veces no me interesa para nada eso, y entonces esos recursos los utilizo de otra manera. Ni siempre me interesan como educadora los recursos que los museos están dando o están promoviendo. Como educadora hay que tratar los museos como tratas los libros, o la información en internet.

LSG: ¿Pero crees qué tenemos suficiente formación como docentes para utilizar esos recursos?

TTE: La formación es a lo largo de la vida. En las carreras profesionales no hay nada de provocación, bueno depende del profesor. Por ejemplo mira lo que hace $\mathrm{M}^{\mathrm{a}}$ Jesús Agra en la Universidad de Santiago, increíble. Creo que con el sistema de Bolonia hay márgenes de trabajo, todo depende de quien lo hace. Depende de lo que tú trasladas a tu alumnado.

LSG: ¿Qué necesita la escuela del museo? ¿Qué puede ofrecer el museo a la escuela? ¿Qué necesita la universidad de los museos y las escuelas y los museos de las escuelas y universidad?

TTE: Yo creo que los museos son equipamientos culturales que la ciudad puede ofrecer a sus ciudadanos y ciudadanas y como tales tienen que saber que son una 
institución pública, unos recursos públicos, unos recursos culturales y lo que las escuelas buscan del museo es eso mismo, un recurso cultural donde puedan ir y hacer estrategias de aprendizaje, es un espacio educativo, tanto desde el punto de vista de la escuela como del punto de vista del museo. Los servicios educativos del museo tienen un concepto de educación completamente diferente del concepto de educación que tiene la escuela. A veces coinciden y no tienen necesariamente porque coincidir, creo que no, cada uno tiene su campo. El museo tiene su campo, un campo significativo de la educación museal, una educación puntual para grupos que van para dar continuidad a temas. Yo creo que es a veces es una educación más "entertainment", "edutainment". Hay muchos museos que tienen la cicatriz de "edutainment". Pero hay museos que yo conozco están realizando actividades muy interesantes, pero son museos con actividades no continuadas sino más bien puntuales, con actividades con grupos de riesgo, con grupos vulnerables, pasa a ser otra cosa, no solo la educación para el museo, pasan a un trabajo más serio. Pero en general, creo que normalmente se realizan actividades puntuales de educación en los museos. A la universidad, los museos solo ofrecen actividades puntuales.

\section{LSG: ¿Qué puede ofrecer la escuela al museo?}

TTE: Si, los profesores no se sienten implicados se sienten externos al proceso, al proceso educativo del museo. Muchas veces yo sé, que hay museos que procuran realizar reuniones con profesorado. Pero creo el problema es que en esas reuniones el profesorado nunca se siente como autor y no se sienten entre iguales, el dialogo que se genera no es un diálogo entre iguales, por eso las escuelas y los profesores no proponen porque se sienten en una situación de inferioridad siempre frente al museo.

\section{LSG: ¿Y con la universidad?}

TTE: Creo que el papel de la Universidad en esa articulación como institución educativa que es, puede articular a las escuelas y museos para promover investigación tanto en el ámbito de la educación como de las ciencias sociales en museos como en escuela, la situación que se puede crear entre escuela y museo y también yo creo que la universidad podría tener un papel más activo, mucho más activo, mediando en los procesos, investigando, abriendo voces. Porque si tuviéramos un tercero mediando las voces sería más fácil crear relaciones más fuertes y articular nuevas estrategias. Porque la universidad además tiene ese papel de investigación, es un movimiento obvio por todos los lados.

\section{LSG: Claro, es que hace falta que se encuentren esas tres instituciones}

TTE: Sí, yo creo que a través de la investigación, sería posible afirmar que el museo y la universidad creen estrategias propias, algunas estrategias para que faciliten este hecho. 


\section{LSG: ¿Debe escolarizarse el museo?}

TTE: No, son diferentes la escuela y el museo, son propósitos culturales y educativos diferentes.

\section{LSG: Sin embargo en algunos museos copian los modelos de las escuelas, los tradicionales}

TTE: Como institución pues se copian modelos de escuela como institución y del hospital también. Tanto escuela como museo continúan con los modelos de los principios de la disciplina. Poco a poco las cosas están abriéndose y comienzan a cambiar.

\section{LSG: ¿Qué crees que aprenden las personas que visitan los museos?}

TTE: Depende, yo creo que pueden no aprender nada, como mucho pueden aprender alguna cosa o mucho. Depende de la apertura que se tenga, pueden aprender sobre sí mismos y puede aprender en el museo sobre los otros, depende de la mirada que se tenga.

LSG: ¿Qué estrategias crees que se pueden poner en marcha para motivar y hacer partícipes al alumnado?

TTE: Pues yo creo que dar voz al alumnado es muy importante y darles un espacio. Darles voz y activar los espacios.

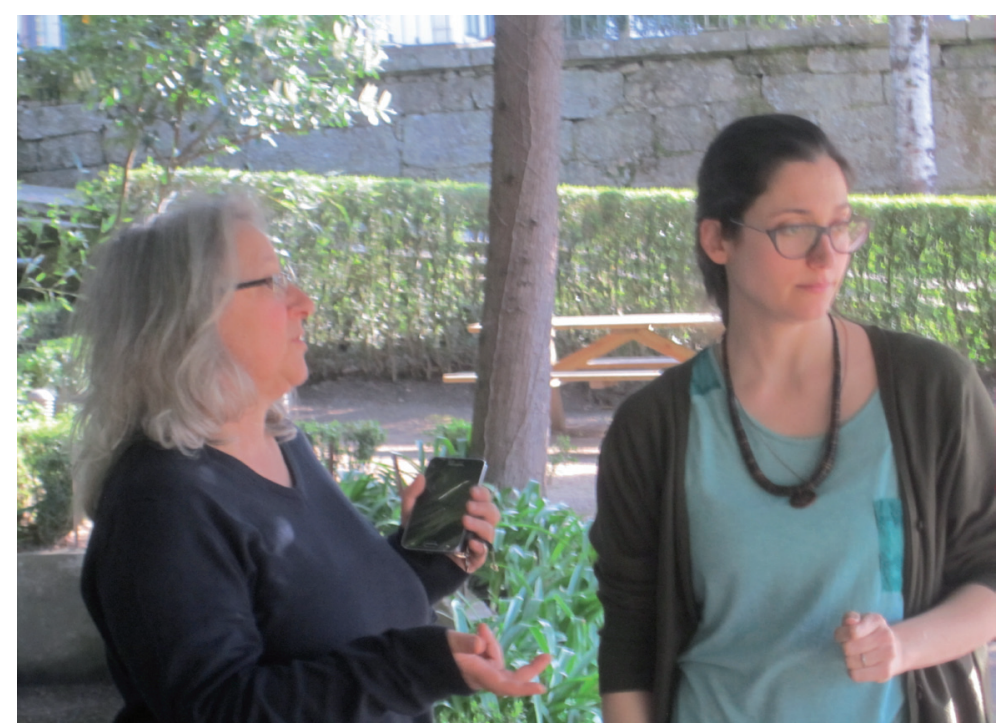

Figura 3. Teresa Torres de Eça presidenta de la AEPV y del INSEA y Rita Santos del Servicio Educativo del Museo Quinta da Cruz (ViseuPortugal), coordinando actividades en el jardín del Museo. 
LSG: Los y las docentes que vienen a los museos ¿qué crees que los mueve? Es decir, ¿Qué incita al profesorado que está en una escuela a venir al museo?

TTE: Pues, hoy en día hay muchos profesores que realizan pocas salidas a los museos porque es muy complicado salir de la escuela en Portugal. Entonces los que salen fuera de la escuela, los que vana los museos es una complicación tremenda. Ir al museo o a cualquier otro sitio, es un observatorio, es importante, es un aprendizaje en contexto real. Y cada vez hay menos.

LSG: ¿Crees que el profesorado que valora esos contextos reales son los que visitan con su alumnado el museo?

TTE: La escuela no valora eso para nada, no valora ese aprendizaje como aprendizaje real, ya que como sabemos que el aprendizaje es saber leer, escribir y contar, no se valora eso como un aprendizaje, no se valora el contexto. Se valora el aprendizaje en el aula, los ejercicios de matemáticas y lo que sale de ahí no se valora.

\section{LSG: Tú crees qué los espacios en los museos habría que repensarlos para sus visitantes, para escolares}

TTE: Hay espacios que necesitan ser repensados en términos de inclusión por ejemplo, en términos de exposición, de "correduría" de exposición. Las exposiciones están hechas solo para adultos, el nivel para trabajar es el nivel de un adulto. Muchos museos no son de fácil acceso para una silla de ruedas, aunque algunos ya tienen hechos unos espacios adaptados.

No solo el espacio del museo, sino el concepto de espacio museal que tiene un status de sagrado y tenemos que cambiarlo de espacio sagrado a espacio púbico. Los propios mediadores tienes que cambiar esa idea. Cuando un grupo accede al museo los "mediadores" tratan el espacio como sagrado, como espacio de respeto, donde no se puede hablar, no se puede correr, no se puede tocar. Entonces yo creo que hay que cambiar la manera de cómo se ve el espacio porque el museo no es un espacio sagrado es un espacio público. Y es de todos, no es del director del museo, no es del director de la exposición. No es de los guías, ni de los políticos. El museo es de la ciudad es del pueblo. En las colecciones privadas si se abren al público hace que sea un espacio público. Yo creo que hay que repensar los espacios, sobre todo hay que repensar como se relacionan las personas con el espacio.

\section{LSG: ¿Tú crees que el tamaño de un museo puede influir en la actividad educativa?}

TTE: No, no. No influye para nada. 


\section{LSG: ¿Cuáles son las herramientas más adecuadas para investigar en educación y museos? ¿Crees qué tenemos que cambiar las estrategias para investigar?}

TTE: La verdad es que todo tipo de investigación es importante, la investigación cuantitativa también lo es. Ahora necesitamos todo, también investigación participativa, investigación acción, investigación a través de la práctica, investigar qué es lo que pasa, llegar a conclusiones y hacer teorías. Investigación participativa donde todos intervengan y que tengan una voz activa tiene que ser más fomentada en este momento que la investigación en el museo todavía es una investigación de estudio de caso, histórica, investigación biográfica. Son siempre investigaciones desde el punto de vista del director, de la historia del museo, de las personas que trabajan en él y muy pocas investigaciones vistas o vividas desde el usuario, de la persona que va al museo. Poca investigación colaborativa y participativa.

Y no es a través de cuestionarios que es lo que tengo visto. Algunas investigaciones utilizan el video, algunas investigaciones utilizan el sujeto como objeto de investigación y no participa para nada, es un objeto de investigación.

\section{LSG: ¿Qué valores y actitudes crees incita el museo? o ¿cuáles debería incitar?}

TTE:Eso es muy difícil. El museo es una institución y cuando nosotros vamos al museo vemos los valores del estado y del poder. Las actitudes que son incitadas, tal vez, son actitudes de cuestionamiento. No sé si siempre será así pero debería haber una actitud de cuestionamiento. ¿Y cuáles debería incitar?, pues creo que el museo debería repensar los espacios y las estrategias porque la carga de poder que tiene no lo puede negar. El museo es una institución del estado, o de una colección privada. Pero nosotros desde las instituciones del poder podemos incitar, nosotros como educadores, podemos crear un tercer espacio de cuestionamiento y emancipación. Yo creo que el museo debería incitar valores críticos de emancipación cultural, de cuestionar y cuestionar, por ejemplo, la relación de poder de la colección. Ser un espacio emancipador, de cuestionamiento, de poner a las personas a pensar sobre sí mismas y sobre los otros.

LS: Se dice que el arte no se enseña, que el arte no se aprende. ¿Estás de acuerdo? ¿Por qué enseñar arte?

TTE: Yo estoy completamente en desacuerdo. Si, si el arte es conocimiento, como decimos que es y pertenece al conocimiento de la humanidad entonces tiene que aprenderse, que desarrollarse y explicarse.

\section{LS: ¿Te gusta enseñar Teresa?}

TTE: Sí me encanta. No tanto enseñar como estar con mis alumnos. Educar es una 


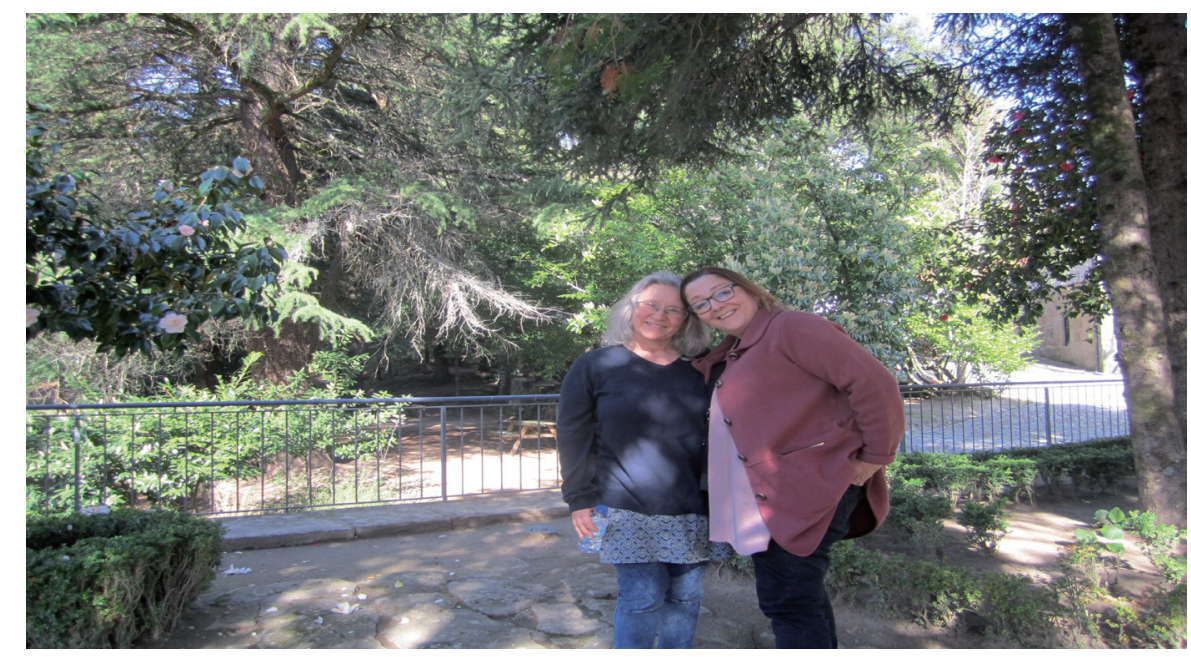

Figura 4. Teresa Torres de Eça y Loli Soto, una vez finalizada la entrevista en los jardines del Museo da Quinta da Cruz (Viseu - Portugal).

palabra con muchas connotaciones y enseñar también. Me gusta mucho la palabra de Paulo Freire que es facilitador. Eso también tiene que ver con la Escuela Moderna, que el profesor es el jardinero. ¿Te acuerdas de esas teorías de educación?, que el profesor tiene que hacer un jardín. Pero no sé, hoy me parece qué como educadora, o sea lo que sea, como artista, hago un trabajo social. Soy una trabajadora social, como artista, como profesora y como investigadora. La artografía como dice Rita Irwin, es una de las metodologías que podemos utilizar, pero hay tantas.

\section{LSG: ¿Qué personas o colectivos han influido más en tu vida?}

TTE: Son muchísimas. Pero puedo decirte que una persona en mi carrera de investigadora muy importante que fue mi director de tesis John Stirs, como director de tesis es como un padre, fue un modelo de investigación, de estar y ver el arte en la educación, no tanto porque siga sus ideas. Fue un modelo para mí como persona. Y mucha más gente, mucha, mucha. Ma Jesús Agra, Rita Irwin,...

\section{LSG: Las cuestiones personales impregnan la trayectoria profesional. ¿Qué cosas destacas como importantes ahora en tu vida?}

TTE: ¿Qué estoy pensando como profesional hoy?, estoy pensando si necesitamos escuela, o cómo la escuela es hoy. La pregunta que me hago muchas veces es para qué la escuela. Si necesitamos escuela y sí la escuela no es también una manera de moldear y de manipular, de eliminar. Pienso que la escuela es una manera de eliminar personas del sistema, pienso muchas veces sobre eso, de la paradoja. La educación enlentecida, esa palabra es de $\mathrm{M}^{\mathrm{a}}$ Jesús, después de una reunión de 
Cadernos Artivistas ${ }^{1}$, trajo la idea de enlentecer y hablamos mucho de ella. Porque lo que estamos perdiéndonos es el tiempo. Hay que retomar el tiempo que nos están robando y que nos estamos robando, cada vez más. Yo trabajo, trabajo, trabajo y no tengo tiempo, es una cuestión personal y profesional, si sigo así también es un suicidio. Todos nosotros que estamos trabajando muchísimo hay que pensar para qué. Y volviendo a la artografía, es muy importante vivir cuestionando. En este momento, las preguntas que me hago todos los días es que tengo que buscar el tiempo, retomar el concepto del tiempo, que se ha perdido. Yo lo he perdido con tanto trabajo. En esa cuestión estoy todos los días, mirándome frente al espejo y decidiéndome bueno Teresa que estás haciendo con tú tiempo. De la calidad, si el tiempo lo más importante. El tiempo, los afectos, pero sin tiempo no hay afectos.

\section{LSG: Gracias Teresa, ha sido un placer y agradezco que destines tu tiempo a compartir de un modo altruista tus pensamientos y opiniones.}

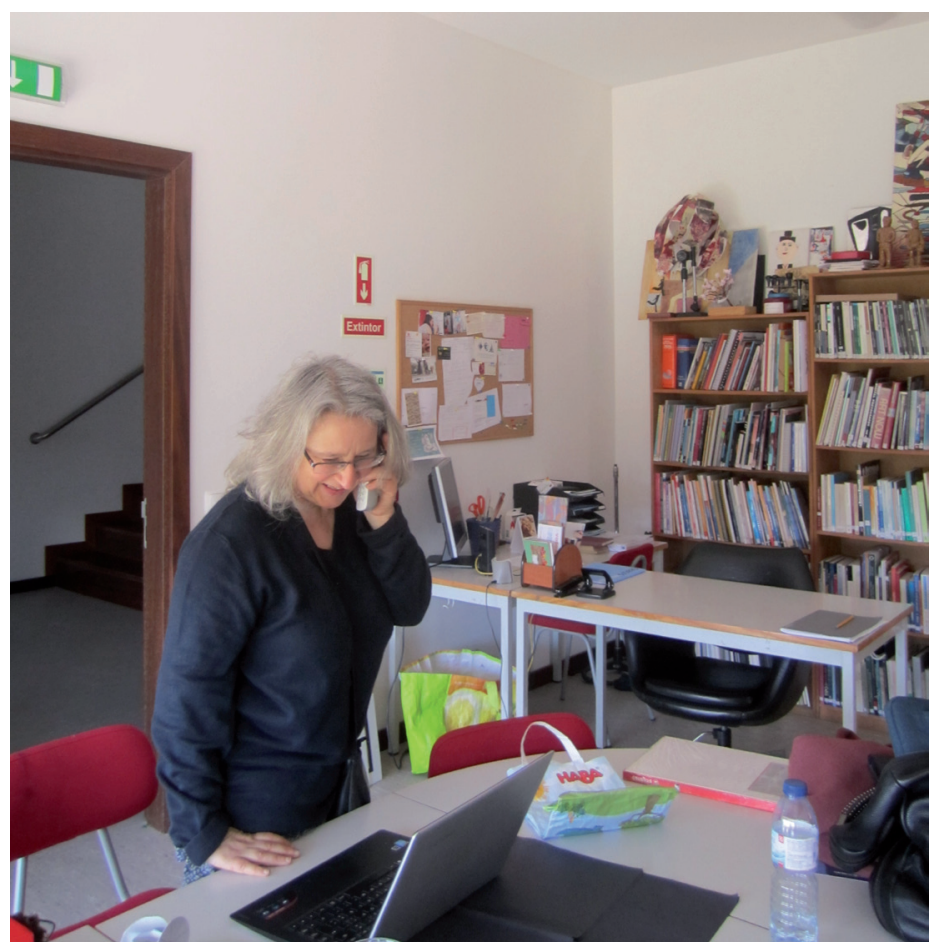

Figura 5. Teresa Torres de Eçaen la sede de AEPV situada en el Museo da Quinta da Cruz (Viseu - Portugal). 